\title{
Impact of polyclonal antithymocyte globulins on kidney transplant outcomes in real-life: a propensity score analysis
}

\section{Jamal Bamoulid}

Centre Hospitalier Universitaire de Besancon

Thomas Crepin

Centre Hospitalier Universitaire de Besancon

\section{Emilie Gaiffe}

Centre Hospitalier Universitaire de Besancon

\section{Bruno Moulin}

Hopitaux universitaires de Strasbourg

\section{Luc Frimat}

Centre Hospitalier Universitaire de Nancy

\section{Philippe Rieu}

Centre Hospitalier Universitaire de Reims

\section{Christiane Mousson}

Centre Hospitalier Universitaire de Dijon

\section{Antoine Durrbach}

Hopital Bicetre

\section{Anne-Elisabeth Heng}

Centre Hospitalier Universitaire de Clermont-Ferrand

\section{Jean-Michel Rebibou}

Centre Hospitalier Universitaire de Dijon

\section{Philippe Saas}

INSERM UMR1098

\section{Cécile Courivaud}

Centre Hospitalier Universitaire de Besancon

\section{Didier Ducloux ( $\nabla$ dducloux@chu-besancon.fr )}

CHU Besancon

\section{Research article}

Keywords: transplantation, polyclonal anti-thymocyte globulins, acute rejection 
Posted Date: August 10th, 2020

DOI: https://doi.org/10.21203/rs.2.18546/v2

License: (c) (i) This work is licensed under a Creative Commons Attribution 4.0 International License. Read Full License 


\section{Abstract}

Background: Randomized studies reported a marginal superiority of polyclonal antithymocyte globulins (ATG, Thymoglobulin ${ }^{\circledR}$, Sanofi, Gentilly, France, or Fresenius ${ }^{\circledR}$, Bad Homburg, Germany) to prevent acute rejection compared to monoclonal anti-CD25 antibodies (IL2Ra). Nevertheless, the representativeness and the generalizability of these studies are questionable.

Methods: We studied the impact of ATG use in real-life conditions in a multicenter study. Propensity score analysis was performed to address potential confounding by indication.

Results: 817 patients were included. Logistic regression revealed that age, male gender, a pre-transplant history of cancer, presence of anti-HLA antibodies, previous kidney transplantation, and transplant center were associated with ATG use. The area under the curve of the propensity score was $0.84+0.02$. ATG use was not associated with a lower rate of acute rejection (18.2\% in ATG-treated patients vs $15.8 \%$ in nonATG-treated patients, $p=0.356)$. Adjustment for propensity score slightly modified the relationship between ATG and acute rejection towards a more neutral effect $(p=0.913)$. Score match analysis recapitulated the previous result. ATG use was associated with the occurrence of opportunistic infection ( $p=0.034)$. There was no difference in graft loss or death between the two groups.

Conclusions: In real-life conditions, ATG does not substantially reduce the risk of acute rejection after kidney transplantation. A better discrimination of patients who may benefit from ATG is required.

\section{Background}

Even when randomized studies are the gold-standard for the evaluation of therapeutic strategies, they are not exempt of potential bias. Indeed, their interpretation has to take into account whether the population is representative of the one receiving the treatment in real life conditions. Moreover, a priori selection of patients by investigators could exclude a fraction of the population meeting inclusion criteria but judged not subject to randomization. Those estimated having the greatest benefit or the highest risks with any treatment arm are likely to be excluded. Additionally, the strict and controlled conditions in which they are conducted, leads to low generalizability because they are performed in very different conditions from real life usual care. Such pitfalls have been identified in different studies (1). This bias is likely concerning evidence-based data concerning ATG. First, ATG use is highly variable from a center to center both as regards the indication and the dose. Second, randomized studies are likely to miss patients with very high immunological risk as well as those carrying severe infectious or neoplastic risks. Another point to consider is time-change modifications of patients' phenotypes and associated treatments that may affect the relevance of past studies. Thus, even when randomized trials are the gold standard, they do have some inconveniences that can be clarified by other methodologic approaches using real-life data.

Two randomized studies reported similar results suggesting that ATG significantly reduced acute rejection risks in high risk patients (2-4). By contrast, the superiority of ATG in low-to-moderate immunological risk patients is more questionable. A large meta-analysis found no benefit of ATG as 
compared to IL2Ra regarding clinically diagnosed acute rejection and a marginal one concerning biopsyproven acute rejection (5). Moreover, whereas the rate of acute rejection is not reduced by ATG use in those patients, infections are more frequent leading to a worse benefit/risk ratio (6-8). Even when these studies have highlighted preferential indications for ATG, its use is highly center-dependent. As a consequence, the global benefit of ATG in real life is more speculative.

We studied the impact of ATG use in real-life conditions in a multicenter study. Propensity score analysis was performed to address potential confounding by indication.

\section{Methods}

\section{Study design and populations}

This study has been conducted in the first 817 consecutive renal transplant recipients (RTR) from the ORLY-EST trial. ORLY-EST is a prospective cohort study including incident RTR in seven French transplant centers (Besançon, Clermont-Ferrand, Dijon, Kremlin-Bicêtre, Nancy, Reims, Strasbourg). The main objective of this study is to assess associations between immune status and post-transplant complications. The ethic committee of Franche-Comté study has approved the study (2008). Patients enrolled in the ORLY-EST study gave their written informed consent.

Among the 817 patients, 265 (32.4\%) had received ATG (Thymoglobulin®, Sanofi, Gentilly, France, or Fresenius ${ }^{\circledR}$, Bad Homburg, Germany) and 552 non-depleting a-CD25 mAb therapy (Basiliximab ${ }^{\circledR}$, Novartis, Basel). Calcineurin inhibitors (CNI [Tacrolimus, $n=514$ (63\%), Cyclosporin, $n=215(31 \%)]$ ) and Mycophenolic Acid ( $\mathrm{n}=784$ (96\%) of which 741 under mycophenolate mofetil) were widely used as immunosuppressive regimen. All the transplants were performed with a negative crossmatch.

Each center uses the anti-Cytomegalovirus (CMV) prevention of their choice. A majority of patients with previous CMV exposition received valganciclovir for 3 months. CMV-naïve patients who received a CMV positive kidney were given valganciclovir for 3 or 6 months. All patients received Pneumocystis antimicrobial prophylaxis with trimethoprim-sulfamethoxazole for at least 6 months.

\section{Confounding factors}

Demographic parameters and medical history were assessed. Dialysis modality and its duration were also recorded. HLA mismatches were registered as well as other relevant immunological parameters such as pre-transplant panel reactive antibodies (PRA) ( 0 vs. positive PRA at any level) and transplant rank (first $v s$. second or more). Delayed graft function defined as the need for dialysis in the first week posttransplant was recorded. Methods of assessment and definitions of these variables have been previously described in detail (9). 


\section{Outcomes}

$\underline{\text { Acute rejection }}$

Only biopsy-proven acute rejections were retained. Acute rejection was defined according to the Banff classification (10).

\section{Severe infections}

Methods to assess infections have been previously described (9).

Briefly, severe bacterial infections or opportunistic infections where only considered if subsequent hospitalization was required. BK infections were not recorded.

\section{$\underline{\text { CMV disease }}$}

CMV disease was defined by the need of treatment in a patient with viral replication.

\section{Statistical analysis}

Median, mean values with standard error, and frequency were provided for continuous and categorical variables, respectively. Medians, means, and proportions were compared using Student's t test and chisquare test (or Fisher's exact test, if appropriate), respectively. Acute rejection free survival was estimated using the Kaplan-Meier method. Follow-up duration was calculated using a reverse Kaplan-Meier estimation. Cox proportional hazard models were performed to estimate hazard ratio (HR) and $95 \% \mathrm{Cl}$ for factors associated with acute rejection.

The association of baseline parameters with acute rejection was first assessed using univariate Cox analyses. Then parameters with $p$ values of less than 0.20 were entered into a final multivariable Cox regression model, after considering collinearity among variables with a correlation matrix. Similar analyses were performed for infections.

In a nonrandomized study, a bias of treatment attribution is always present. Differences between the two groups due to ATG indication may greatly influence the outcomes. To face these constraints, we calculated propensity scores to correctly adjust for confounding factors and to address potential confounding by indication (11).

We assessed the propensity score of receiving ATG through multivariable logistic regression model with ATG use as the dependent variable. Data known to influence the choice of using or not ATG were used to 
calculate the propensity score and included age, pretransplant history of malignancy, duration of dialysis before transplant, pre-transplant anti-HLA antibodies, previous history of transplantation, Other factors such as sex, previous cardiovascular disease, hypercholesterolemia, hypertension, body mass index, smoking status, and diabetes, were also tested in the model. We matched one ATG-treated patient to one non-ATG-treated patient by their propensity score $+0.02(0.10 S D$ of the propensity score) to generate a sub-cohort of 154 patients who were treated with ATG and 154 patients who were not.

\section{Results}

Study_population

Characteristics of the study population were depicted in table 1.

\section{Determinants of ATG use}

In univariate analysis, age $(p=0.007)$, gender $(p=0.003)$, a previous history of cancer $(p=0.011)$, presence of HLA antibodies $(p<0.001)$, previous kidney transplantation $(p<0.001)$, transplant center $(p<0.001)$, dialysis duration $(p<0.001)$, body mass index $(p=0.038)$, previous exposition to CMV $(p=0.001)$, and delayed graft function $(p=0.001)$ were associated with ATG use.

Logistic regression revealed that age $(\mathrm{HR}, 0.98 ; 95 \% \mathrm{Cl}, 0.96$ to 0.99 for each increase of one-year in age; $p=0.005)$, male gender $(\mathrm{HR}, 0.69 ; 95 \% \mathrm{Cl}, 0.45$ to $0.96 ; p=0.041)$, a pre-transplant history of cancer (HR, $0.21 ; 95 \% \mathrm{Cl}, 0.07$ to $0.64 ; p=0.006)$, presence of anti-HLA antibodies (HR, 5.90; $95 \% \mathrm{Cl}, 3.71$ to 9.38; $p<0.001)$, previous kidney transplantation $(\mathrm{HR}, 5.81 ; 95 \% \mathrm{Cl}, 2.42$ to $13.98 ; p<0.001)$, and transplant center $(p<0.001)$ (table) were associated with ATG use.

On the basis of this model, a propensity score was assessed for each patient (mean 0.32 \pm 0.26 ; range, $0.002-0.970$ ). The area under the curve of the propensity score was $0.84 \pm 0.02$. Excluding center effect significantly reduced the predictive value of the model (AUC $0.77 \pm 0.02$ vs $0.84 \pm 0.02 ; p<0.001$ ).

Propensity score matching successfully balanced the distribution of characteristics across patients who were treated with ATG compared with those who were not (table 2). Mean propensity scores were similar in patients who experienced acute rejection compared with those who did not $(0.37 \pm 0.19)$.

\section{Acute rejection}

One hundred and forty-eight patients (18\%) experienced at least one episode of acute rejection. Most of them have T cell mediated acute rejection (TCMR) (138, 93\%).

Previous kidney transplantation $(\mathrm{HR}, 0.69 ; 95 \% \mathrm{Cl}, 0.45$ to $0.96 ; p=0.041)$ and delayed graft function (HR, $0.69 ; 95 \% \mathrm{Cl}, 0.45$ to $0.96 ; p=0.041$ ) were the only factors associated with the occurrence of acute rejection. Same results were obtained when restricting the analysis to TCMR. 
ATG use was not associated with a lower rate of acute rejection (18.2\% in ATG-treated patients vs $15.8 \%$ in non-ATG-treated patients; $\mathrm{HR}, 1.18 ; 95 \% \mathrm{Cl}, 0.83$ to $1.69 ; p=0.356$ ) (figure 1 and table 3 ). No protective effect was observed in any subgroups of patients (table 3 ) nor when analysis was restricted to TCMR.

Adjustment for propensity score slightly modified the relationship between ATG and acute rejection towards a more neutral effect $(\mathrm{HR}, 1.02 ; 95 \% \mathrm{Cl}, 0.66$ to $1.58 ; p=0.913)$. Score match analysis recapitulated the previous result.

\section{Infections and ATG use}

\section{Severe bacterial infection}

Two hundred and sixty-two patients (32\%) experienced at least one episode of severe bacterial infection. Age $(p=0.007)$, gender $(p=0.022)$ body mass index $(p=0.008)$, pre-transplant diabetes $(p=0.004)$, and Tacrolimus use $(p=0.081)$ were associated with SBI.

Cox analysis revealed that age $(\mathrm{HR}, 1.02 ; 95 \% \mathrm{Cl}, 1.01$ to 1.03 for each increase of one-year in age; $p=0.005)$, male gender $(\mathrm{HR}, 0.70 ; 95 \% \mathrm{Cl}, 0.52$ to $0.95 ; p=0.022)$, body mass index $(\mathrm{HR}, 1.06 ; 95 \% \mathrm{Cl}, 1.03$ to 1.09 for each increase of $1 \mathrm{~kg} / \mathrm{m}^{2}$ in $\left.\mathrm{BMl} ; p<0.001\right)$, and Tacrolimus use (HR, 1.38; $95 \% \mathrm{Cl}, 1.01$ to 1.92 ; $p=0.048$ ) (table) were associated with SBI.

ATG use was not associated with a higher rate of SBI (HR, 0.84; 95\%Cl, 0.61 to 1.16; $p=0.290)$. Adjustment for propensity score did not modify the relationship between ATG and SBI $(\mathrm{HR}, 0.86 ; 95 \% \mathrm{Cl}$, 0.58 to $1.28 ; p=0.467)$.

\section{Opportunistic infections}

Seventy three patients (9\%) experienced at least one episode of opportunistic infection (Zoster, 24; Herpes, 14; Pneumocystis Jerovicii, 12; Legionella, 8; Tuberculosis, 7; Aspergillosis, 3; Nocardiosis, 3; Mucormycosis, 1 ; Cryptococcal infection, 1). ATG use was the only predictive factor for opportunistic infection ( $\mathrm{HR}, 1.77 ; 95 \% \mathrm{Cl}, 1.26$ to $2.70 ; p=0.008)$. ATG use remained associated with the occurrence of opportunistic infection after adjustment for propensity score (HR, 1.55; 95\% Cl, 1.12 to $2.41 ; p=0.034)$

\section{$\underline{\text { CMV disease }}$}

One hundred and thirty-one patients (16\%) experienced CMV disease. CMV exposure $(p=0.002)$, anti-CMV prophylaxis $(p<0.001)$, and ATG use $(p=0.018)$ were associated with CMV disease. Nevertheless, ATG use was no more associated with the occurrence of CMV disease after adjustment for propensity score (HR, $0.89 ; 95 \% \mathrm{Cl}, 0.55$ to $1.44 ; \mathrm{p}=0.642)$.

\section{Death and graft loss}

Twenty-four patients (3\%) died in the first year post-transplant. There was no difference in the two groups (3\% in ATG-treated patients and $2.9 \%$ in those having received IL2Ra; $p=0.924$ ). 
Thirty-eight patients (4.7\%) lost their graft in the first year post-transplant. There was no difference in graft loss between the two groups (4.5\% in ATG-treated patients and $4.7 \%$ in those having received IL2Ra; $\mathrm{p}=0.908)$.

\section{Discussion}

T cell depletion by polyclonal anti-thymocyte globulins (ATG) has been used for many years for immunosuppression in organ transplantation. These products are composed of polyclonal antibodies mainly directed against $\mathrm{T}$ lymphocytes, but also against other types of immune and non-immune cells, such as stromal thymic cells $(12,13)$. Although they have been widely used for over 30 years, few evidence-based guidelines for ATG use are available and prescription remains largely empirical. A substantial benefit of ATG has been described only in immunological high-risk patients. However, a significant part of ATG-treated patients included in this study could not be classified as immunological high-risk patients according to traditional definitions. One in five patients having received ATG was the recipient of a first transplant and had no HLA sensitization. However, $12 \%$ of HLA-sensitized recipients of a second transplant received IL2Ra induction.

We found some factors, usually not used as inclusion or exclusion criteria in randomized studies comparing ATG and a-CD25, to significantly influence the choice of induction treatment. A pre-transplant history of cancer was strongly associated with avoidance of ATG. A large meta-analysis reported an increased incidence of cancer in ATG-treated patients as compared to those having received IL2Ra (4). Our group also emphasized the impact of ATG-induced persistent CD4 lymphopenia on post-transplant cancer occurrence $(14,15)$. It is likely that, not only an immunological risk, but also a putative enhanced risk of cancer participates in the final choice of the type of induction. While only $7 \%$ of patients were 70 years old or over, older age significantly prevents ATG use. However, cut off was 70 or 75 years old in the different randomized studies comparing ATG to IL2Ra (5). A supposed frailty of immune responses in elderly probably explains this association. Finally, while no data supports a specific benefit of ATG in female, male were less prone to receive ATG than female. Apart from these parameters, an unexplainable proportion of the decision is center-dependent and reflects individual practice. Furthermore, the use of ATG was not associated with any different strategy of maintenance immunosuppression. Scheduled corticosteroid withdrawal was similar in the two treatments groups as well as the use of Tacrolimus or Mycophenolic acid. This suggests that the choice to use ATG is based on both evidence- and belief-based criteria or that evidence-based data are not sufficiently convincing to bring together physicians' opinions.

Our study suggests that, in real life conditions, ATG use is not associated with reduced rates of acute rejection. This result was observed in all sub-groups of patients, including those who have been identified as having greater benefits in randomized studies $(2,3)$. Because the majority of patients included in this study were at low-to-intermediate immunological risk, a lack of statistical power may explain this result. 
Nevertheless, no trends were observed suggesting that enhancing power would not change the results. Some explanations are possible. Randomized studies have been published almost ten years ago and more. Better determination of HLA antibodies and generalization of flow cytometry crossmatch may have improved donor-recipient compatibility and reduce the risk of acute rejection. As a consequence, the beneficial effects of ATG may be reduced. According to this hypothesis, HLA sensitization was not associated with acute rejection even in patients with PRA $>30 \%$. The two only predictive factors of acute rejection were a previous history of kidney transplantation and delayed graft function. We observed a non-significant reduction of $19 \%$ in the risk of acute rejection after delayed graft function in ATG-treated patients. As a result, a randomized study should include 778 patients to demonstrate such a significant difference with a power of $80 \%$ and an alpha risk of $5 \%$. Our study highly suggests that ATG did not reduce the rate of acute rejection in current conditions of utilization.

Even when CMV disease was more frequent in ATG-treated patients, the association did not persist after adjustment for confounding factors. Furthermore, due to different center practices, CMV prophylaxis was surprisingly less frequent in ATG-treated patients. According to propensity score analysis, this confirms that CMV status did not influence the choice to use ATG. Under systematic CMV prophylaxis, the rate of CMV disease are relatively similar in ATG-treated and non-ATG-treated patients $(2,8)$. By contrast, in the absence of CMV prophylaxis, CMV disease was probably much more frequent in patients having received ATG $(5,16)$. No difference in the incidence of severe bacterial infection was observed between the two arm groups. Such a result is consistent with previous reports (3). However, we observed more frequent opportunistic infections in ATG-treated patients. This result confirms preliminary analyses performed earlier in this study (17). Overall, ATG is not associated with a substantial increase in early infectious complications except for opportunistic infections. Furthermore, both patient and graft survivals were similar in the two treatment groups.

Our study has some limitations. A bias of indication is inherent to non-randomized comparisons of treatment. We used propensity score analysis to overcome this limitation. Patients who received ATG differed in many ways from those who did not. Propensity score analysis takes these differences into account and minimizes potential confounding by indication. After adjustment for propensity score, we did not observe any difference in most post-transplant outcomes between patients having or not having received ATG. Registry studies have the potential to include a larger number of patients, but often lack accurate confounding factors analysis. Our study is likely to be a fair compromise between less precise observational registers and unfeasible randomized trials. Due to the prospective design of the study, missing data are low $(<5 \%)$.

\section{Conclusion}


Our study suggests that, in real-life conditions, ATG does not substantially reduce the risk of acute rejection after kidney transplantation. By contrast, ATG-treated patients exhibited an increased risk of opportunistic infections. A better discrimination of patients who may benefit of ATG is required.

\section{Declaration}

\section{Ethics approval and consent to participate}

Sample collection was performed after regulatory approval by the French ministry of health (agreement number \# DC-2008-713, June 11 ${ }^{\text {th }}$ 2009). The ethic committee of Franche-Comté study has approved the study (2008). Patients enrolled in the ORLY-EST study gave their written informed consent. Clinical data were prospectively collected.

\section{Consent for publication}

Not applicable

\section{Availability of data and materials}

The datasets used and/or analyzed during the current study are available from the corresponding author on reasonable request.

\section{Competing interest}

The authors have no competing interest

\section{Fundings}

Supported by grants from the Fondation Transplantation, the PHRC 2005 and 2011 (to DD), the DHOS/INSERM/INCa (Appel d'offre Recherche Translationnelle 2008 to DD) and the APICHU 2010 (to JB), from the Agence Nationale de la Recherche (Labex LipSTIC, ANR-11-LABX-0021) and the Région de Franche-Comté (support to Labex LipSTIC to PS). JB, CC, TC, and CR received financial support from the Fondation Transplantation (\#ET-031211 and \#ET-050320, respectively). This work is a part of the RIALTO (Research in Immunology of AtheroscLerosis after TransplantatiOn) program. This work is supported by the Fédération hospitalo-universitaire INCREASE (INtegrated Centre for Research in Inflammatory DisEASEs). 
Funding organizations had no access on the design of the study, and collection, analysis, and interpretation of data

\section{Acknowledgements}

Not applicable

\section{Authors' contributions}

JB, CC, TC, DD, JMR, and PS designed the study concept and drafted the manuscript. DD, TC, CC, EG, CC, $J B, B M, L F, P R, C M, A D$ and $A E H$ participated to acquisition of data and patient follow up. DD did statistical analysis. All authors saw and approved the final version of the manuscript. All the authors approved the final manuscript.

\section{References}

1- Britton A, McKee M, Black N, McPherson K, Sanderson C, Bain C. Threats to applicability of randomised trials. Exclusions and selective participation. J Health Serv Res Policy 1999; 4: 112-21.

2- Brennan DC, Daller JA, Lake KD, Cibrik D, Del Castillo D; Thymoglobulin Induction Study Group: Rabbit antithymocyte globulin versus basiliximab in renal transplantation. N Engl J Med 355: 1967-1977, 2006

3- Noël C, Abramowicz D, Durand D, Mourad G, Lang P, Kessler M, Charpentier B, Touchard G, Berthoux F, Merville P, Ouali N, Squifflet JP, Bayle F, Wissing KM, Hazzan M: Daclizumab versus antithymocyte globulin in high-immunological-risk renal transplant recipients. J Am Soc Nephrol 20: 1385-1392, 2009

4- Alloway RR, Woodle ES, Abramowicz D, Segev DL, Castan R, Ilsley JN, Jeschke K, Somerville KT, Brennan DC. Rabbit anti-thymocyte globulin for the prevention of acute rejection in kidney transplantation. Am J Transplant 19: 2252-61, 2019

5- Webster AC, Ruster LP, McGee RG, Matheson SL, Higgins GY, Willis NS, Chapman JR, Craig JC. Interleukin 2 receptor antagonists for kidney transplant recipients. Cochrane Database Syst Rev 2010; CD003897

6- Mourad G, Garrigue V, Squifflet JP, Besse T, Berthoux F, Alamartine E, Durand D, Rostaing L, Lang P, Baron C, Glotz D, Antoine C, Vialtel P, Romanet T, Lebranchu Y,AlNajjarA,Hiesse C, Potaux L, Merville P, Touraine JL, Lefrancois N, Kessler M, Renoult E, Pouteil-Noble C, Cahen R, Legendre C,Bedrossian J, LePogampP, Rivalan J,Olmer M, Purgus R,Mignon F,Viron B, Charpentier B: Induction versus non-induction in renal transplant recipients with tacrolimus-basedimmunosuppression. Transplantation 72: 1050-1055, 2001 
7- Lebranchu Y, Bridoux F, Büchler M, Le Meur Y, Etienne I, Toupance O, Hurault de Ligny B, Touchard G, Moulin B, Le Pogamp P, Reigneau O, Guignard M, Rifle G: Immunoprophylaxis with basiliximab compared with antithymocyte globulin in renal transplant patients receivingMMFcontaining triple therapy. Am J Transplant 2: 48-56, 2002

8- Sollinger H, KaplanB, PescovitzMD, PhilosopheB, RozaA, BraymanK, Somberg K: Basiliximab versus antithymocyte globulin for prevention of acute renal allograft rejection. Transplantation 72: 1915-1919, 2001

9- Crepin T, carron C, Roubiou C, Gaugler B, Gaiffe E, Simula-Faivre D, Ferrand C, Tiberghien P, Chalopin JM, Moulin B, Frimat L, Rieu P, Saas P, Ducloux D, Bamoulid J. ATG-induced immune senescence: clinical implications in renal transplant recipients. Am J Transplant 2015; 15: 1028-38

10- Solez $\mathrm{K}$, Axelsen RA, Benediktsson $\mathrm{H}$, et al. International standardization of criteria for the histologic diagnosis of allograft rejection: The Banff working classification of kidney transplant pathology. Kidney Int 1993; 44: 411-422

11- Joffe MM, Rosenbaum PR: Invited commentary: Propensity scores. Am J Epidemiol 150: 327-333, 1999

12- Rebellato LM, Gross U, Verbanac KM, Thomas JM. A comprehensive definition of the major antibody specificities in polyclonal rabbit antithymocyte globulin. Transplantation. 1994;57(5):685-694.

13- Bonnefoy-Berard N, Vincent C, Revillard JP. Antibodies against functional leukocyte surface molecules in polyclonal antilymphocyte and antithymocyte globulins. Transplantation. 1991;51(3):669-673.

14- Ducloux D, Courivaud C, Bamoulid J, Vivet B, Chabroux A, Deschamps M, Rebibou JM, Ferrand C, Chalopin JM, Tiberghien P, Saas P. Prolonged CD4 T cell lymphopenia increases morbidity and mortality after renal transplantation. J Am Soc Nephrol. 2010;21: 868-75

15- Ducloux D, Carron PL, Rebibou JM, Aubin F, Fournier V, Bresson-Vautrin C, Blanc D, Humbert P, Chalopin JM. CD4 lymphocytopenia as a risk factor for skin cancers in renal transplant recipients. Transplantation. 1998; 65: 1270-2.

16- Luan FL, Samaniego M, Kommareddi M, Park JM, Ojo AO. Choice of induction regimens on the risk of cytomegalovirus infection in donor-positive and recipient-negative kidney transplant recipients. Transpl Infect Dis. 2010 Dec;12(6):473-9

17- Crepin T, Gaiffe E, Courivaud C, Roubiou C, Laheurte C, Moulin B, Frimat L, Rieu P, Mousson C, Durrbach A, Heng AE, Saas P, Bamoulid J, Ducloux D. Pre-transplant end-stage renal disease-related immune risk profile in kidney transplant recipients predicts post-transplant infections. Transpl Infect Dis. 2016; 18: 415-22 


\section{Tables}

Page 13/21 
Table 1: Demographic characteristics of the study patients according to ATG treatment

\begin{tabular}{|c|c|c|c|}
\hline & $\begin{array}{c}\text { ATG-treated patients } \\
(\mathbf{n = 2 6 5 )}\end{array}$ & $\begin{array}{c}\text { Non ATG-treated patients } \\
(\mathbf{n}=552)\end{array}$ & $\mathbf{p}$ \\
\hline Age (years) & $53 \pm 13$ & $50 \pm 13$ & 0.001 \\
\hline Gender (\% male) & $56 \%$ & $67 \%$ & 0.003 \\
\hline Dialysis (\%) & $93 \%$ & $91 \%$ & 0.332 \\
\hline Dialysis duration (months) & $47+41$ & $35+27$ & $<0.001$ \\
\hline Pre-transplant diabetes & $22 \%$ & $18 \%$ & 0.269 \\
\hline Pre-transplant history of cancer & $3 \%$ & $7 \%$ & 0.011 \\
\hline Body mass index (\%) & $25 \pm 4.9$ & $4 \%$ & 0.038 \\
\hline Previous transplantation & $19 \%$ & $11 \%$ & $<0.001$ \\
\hline Living donor (\%) & $9 \%$ & $19 \%$ & 0.382 \\
\hline PRA > 0 (\%) & $59 \%$ & $9 \%$ & $<0.001$ \\
\hline PRA > 30\% & $25 \%$ & $52 \%$ & $<0.001$ \\
\hline CMV exposure (\%) & $64 \%$ & $57 \%$ & 0.001 \\
\hline CMV prophylaxis (\%) & $45 \%$ & $65 \%$ & 0.003 \\
\hline Tacrolimus (\%) & $59 \%$ & $97 \%$ & 0.203 \\
\hline MPA (\%) & $97 \%$ & $9.3 \%$ & 0.872 \\
\hline (\%) & $8.8 \%$ & & 0.794 \\
\hline Scheduled corticoid withdrawal & $30 \%$ & $21 \%$ & 0.001 \\
\hline Delayed graft function (\%) & & & \\
\hline
\end{tabular}


Page 15/21 
Page 16/21 
Table 2: Demographic characteristics of the propensity score study cohort

\begin{tabular}{|c|c|c|c|}
\hline & $\begin{array}{c}\text { ATG-treated patients } \\
(\mathbf{n = 1 5 4 )}\end{array}$ & $\begin{array}{c}\text { Non ATG-treated patients } \\
(\mathrm{n}=154)\end{array}$ & $\mathbf{p}$ \\
\hline Age (years) & $50 \pm 13$ & $50 \pm 14$ & 0.829 \\
\hline Gender (\% male) & $61 \%$ & $59 \%$ & 0.727 \\
\hline Dialysis (\%) & $92 \%$ & $92 \%$ & 1 \\
\hline Dialysis duration (months) & $49 \pm 44$ & $42 \pm 35$ & 0.775 \\
\hline Pre-transplant diabetes & $21 \%$ & $40 \%$ & 0.842 \\
\hline Pre-transplant history of cancer & $3 \%$ & $24.4 \pm 4.2$ & 0.759 \\
\hline Body mass index (\%) & $24.9 \pm 4.9$ & $6 \%$ & 0.384 \\
\hline Previous transplantation & $7 \%$ & $9 \%$ & 0.788 \\
\hline Living donor (\%) & $9 \%$ & $37 \%$ & 1 \\
\hline PRA > 0 (\%) & $38 \%$ & $15 \%$ & 0.898 \\
\hline PRA > 30\% & $15 \%$ & $57 \%$ & 1 \\
\hline Pre-transplant CMV exposure (\%) & $55 \%$ & $57 \%$ & 0.732 \\
\hline CMV prophylaxis (\%) & $45 \%$ & $63 \%$ & 0.125 \\
\hline Tacrolimus (\%) & $55 \%$ & $98 \%$ & 0.164 \\
\hline MPA (\%) & $98 \%$ & $8.5 \%$ & 1 \\
\hline Scheduled corticoid withdrawal & $9.6 \%$ & & 0.692 \\
\hline (\%) & & $24 \%$ & 0.248 \\
\hline Delayed graft function (\%) & $30 \%$ & & \\
\hline
\end{tabular}


Page 18/21 
Page 19/21 
Table 3: Hazards ratio of ATG for acute rejection (unadjusted and adjusted for relevant parameters in the overall cohort and in different subgroups)

Unadjusted, 1.18 95\%CI, 0.83 to $1.69 ; p=0.356$

Adjusted for,

Age, $1.1895 \%$ CI, 0.83 to $1.70 ; p=0.345$

Gender, 1.22 95\%CI, 0.86 to $1.75 ; p=0.269$

Previous transplantation, 1.09 95\%CI, 0.75 to $1.58 ; p=0.649$

Presence of HLA antibodies, $1.1795 \%$ CI, 0.79 to $1.73 ; p=0.356$

Delayed graft function, $1.1595 \% \mathrm{CI}, 0.78$ to $1.64 ; p=0.466$

Center, 1.08 95\%CI, 0.74 to $1.59 ; p=0.682$

Propensity score, 1.02 95\%CI, 0.66 to $1.58 ; p=0.913$

\section{Figures}

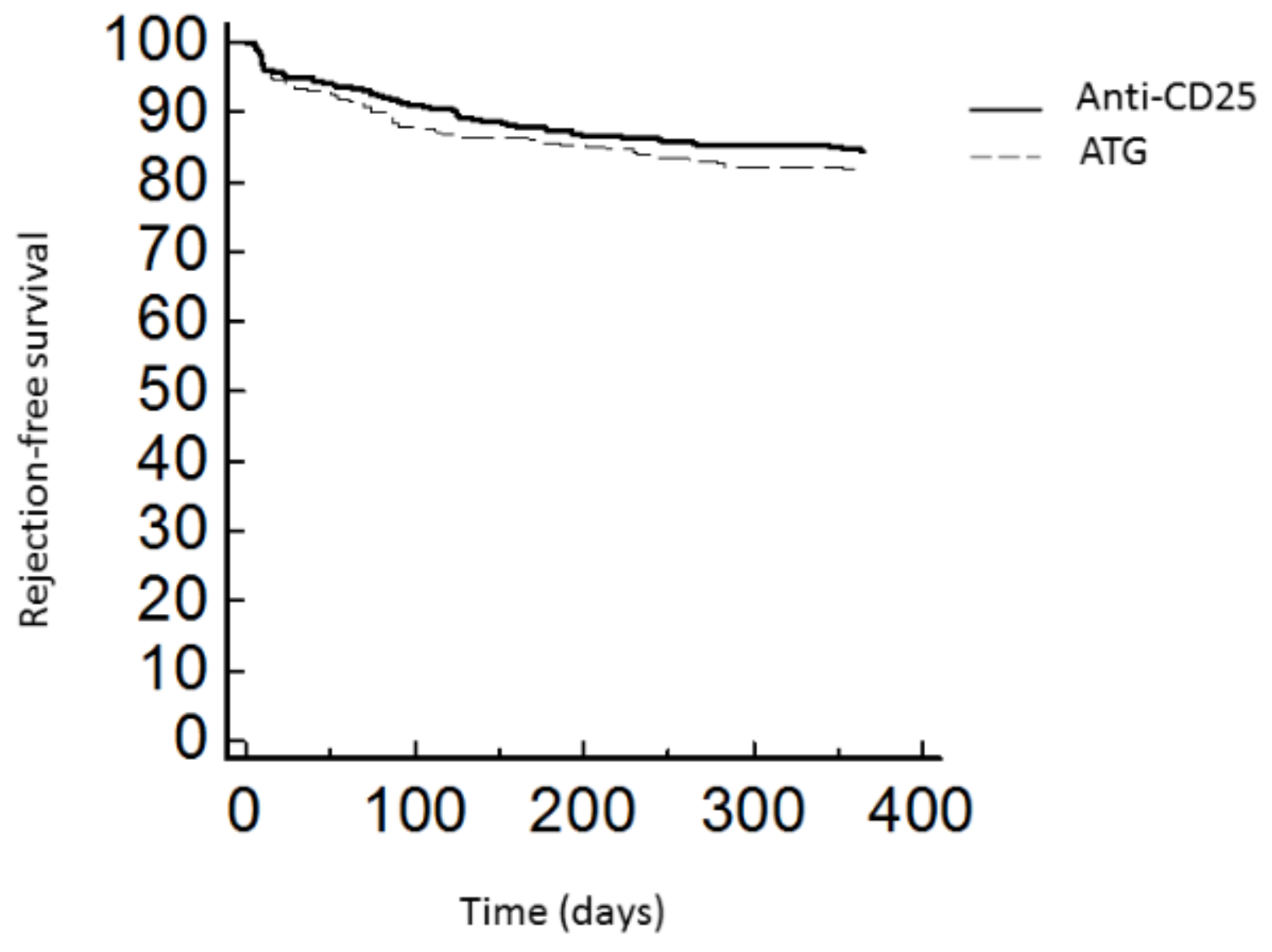

Figure 1 
Kaplan-Meier acute rejection-free survival curves for patients according to the use of ATG

\section{Supplementary Files}

This is a list of supplementary files associated with this preprint. Click to download.

- SUPPLEMENTARYFILE.docx 